\title{
QUALITATIVE AND QUANTITATIVE CHANGES IN THE CELLULAR COMPOSITION OF GUMS IN PATIETS WITH PERIODONTAL DISEASES
}

\author{
N. V. Hasiuk \\ I. HORBACHEVSKY TERNOPIL STATE MEDICAL UNIVERSITY, TERNOPIL, UKRAINE
}

Background. The results of a comprehensive study of cytological features of epithelial differentiation processes in gums in patients with generalized periodontitis are presented in the article.

Objective. The objects of clinical observations were patients with generalized periodontitis. The disease duration was 3-5 years.

Methods. Material for the study was gums epithelium taken from a marginal area of gums in young adults, with generalized periodontitis. The epithelium was removed with a spatula, with subsequent transfer to a glass plate and drying in the open air for 3-5 minutes. The colouring of the material was done according to RomanovskyyHimza, with a next microscopic and morphological analysis, taking into account the percentage of different forms of epithelial cells in normal and due to an age aspect.

Results. One of cytological signs of periodontal pathology in young people is epithelial cells with signs of irritation. It should be noted that these cellular elements belong to the intermediate layer of cells that meet histological sections and ribbed surface layers are characterized by homogenization and vacuolization of cytoplasm as a manifestation of prolonged irritation by bacterial cell aggression.

Conclusions. Analysis of cytograms proved general periodontitis in the examined patients, in absence of adequate therapy as automotive continuous process. Its components may regress under the influence of effective treatment and re-activate during exacerbation of the inflammatory process under the influence of pathogenic factors such as microbial, hygienic and immune.

KEY WORDS: gingiva, epithelium, cells, nucleus, cytoplasm.

\section{Introduction}

As a part of the mucous membrane, buccal epithelium is active within irritating factors that influence it from external and internal environment. It allows to use it for studying the physiology and reactivity of mucous membranes, and in particular as an indicator of local and general disorders of homeostasis [1, 2].

Each anatomical area of gums has a zonal type of keratinization and on histological sections is characterized by basal, ribbed, granular and stratum layers; keratinization occurs in it due to ortokeratoz - a phased process, and as well due to apoptotic changes in the upper layer of epithelium [3].

An only exception is gum sulcus epithelium that is not keratinized and reduces its barrier

Corresponding author: Natalya Hasiuk, Department of Therapeutic Dentistry, I. Horbachevsky Ternopil State Medical University, 1 Maidan Voli, Ternopil, Ukraine, 46001

Phone number: +380352259802

E-mail: gasyknv@tdmu.edu.ua function and is a target organ at the stage of periodontal pathology development [4].

Taken into account the spread of general periodontitis among young people [5] and the prevailing current points of view about the disease pathogenesis $[6,7,8]$, the processes of differentiation of gums epithelial cells in the course of general periodontitis is an urgent matter, as well as diagnosis objectification, determination of severity degree and involvement of adjacent connective tissue to predict course of the disease, exacerbation rate and relapse $[9,10,11,12,13]$.

Our study was aimed to determine the characteristic features of cellular structure of gums cytogram in patients with general periodontitis.

\section{Material and Methods}

Material for the study was gums epithelium, taken from a marginal area of gums in young 
adults, with generalized periodontitis. The disease duration was 3-5 years.

The epithelium was removed with a spatula, with subsequent transfer to a glass plate and drying in the open air access for 3-5 minutes. The colour of the material was done according to Romanovskyy-Himza, with a next microscopic and morphological analysis, taking into account the percentage of different forms of epithelial cells in norm and due to an age aspect.

In order to standardize the epithelium layer and a deeper studying, in our research we used cytological classification; according to it basal, parabasal, intermediate, superficial cells were in the epithelium of gum in the area of keratinized epithelium of gums, horny scales.

Statistical analysis of the results was done at the Department of Statistical Research of I. Horbachevsky Ternopil State Medical University. Parametric methods were used for the indicators, their distribution met general requirements.

To evaluate the nature of distribution the coefficients of skewness and kurtosis were determined. The test was performed with normal asymmetry test by Shapiro-Wilkie. Probable differences of the results for different groups were determined using Student t-test.

The difference was considered likely in common in the medical and research error probability $p<0.05$. The probability of error was evaluated by the Student t-test tables due to the size of the experimental groups. The rate of distribution was statistically significantly different from the expected normal nonparametric criterion (U) Mann-Whitney nonparametric analogue of a Student t-test.

\section{Results}

It is established that a major component of the cellular composition of the gums' scraping cytogram in the course of generalized periodontitis are the stratified squamous epithelial cells. They are present in cytograms under normal conditions and during pathology. It should be emphasised that the squamous cells are heterogeneous, reflecting the heterogeneity of epithelial cells gums' cover. In the case of generalized periodontitis a cytological character of scrap changes significantly both in epithelial and connective tissue constituents.

Detailed statistical analysis of cytogram, made it possible to determine the percentage change in gingival epithelial cells of people examined due to the different severity of generalized periodontitis.

This ratio is $(2,20 \pm 0,10):(9,30 \pm 0,16)$ : $(41,6 \pm 0,36):(37,0 \pm 0,31):(9,9 \pm 0,16)$ (Table 1).

\section{Discussion}

The qualitative and quantitative cellular composition of cytogram in periodontitis depends on the clinical course and intensity of inflammatory and degenerative processes in periodontal tissues. The received data was significantly different from the stereotypical percentage of epithelial differentiation of stratified squamous epithelium of gums [3], and ratio that was determined for the examined patients and is characterized by violation of differentiation: disorder of keratinization as evidenced by the presence of all components of different epithelial cells.

This makes it possible to assert that during generalised periodontitis the mechanisms of epithelial desquamation disruption take place causing barrier function of gums at generalized periodontitis; that is the outcome of different epithelial cells differentiation caused by inflammatory infiltrate of lamina propria.

The presence of parabasal cells in cytograms was explained by activation of compensatory mechanisms of epithelium, taken into account high mitotic activity of gum epithelium and increased glycogen-containing epithelial cells.

Table 1. Percentage change in different cells classes of stratified squamous gums epithelium in the course of generalized periodontitis

\begin{tabular}{|l|c|c|c|c|c|}
\hline \multirow{2}{*}{ Indicator } & \multicolumn{5}{|c|}{ Cells of smears } \\
\cline { 2 - 6 } & Basic & Parabasal & Intermediate & Superficial & Horny scales \\
\hline $\begin{array}{l}\text { Norm } \\
\text { (V.L. Bykov) }\end{array}$ & 0 & 0 & 60 & 8 & 32 \\
\hline $\begin{array}{l}\text { Norm for young } \\
\text { person }\end{array}$ & 0 & 0 & $59,20 \pm 1,15^{*}$ & $7,60 \pm 0,34^{*}$ & $33,2 \pm 0,65^{*}$ \\
\hline $\begin{array}{l}\text { Generalized } \\
\text { periodontitis }\end{array}$ & $2,20 \pm 0,10^{* *}$ & $9,30 \pm 0,16^{* *}$ & $41,60 \pm 0,36^{* *}$ & $37,0 \pm 0,31^{* *}$ & $9,90 \pm 0,16^{* *}$ \\
\hline
\end{tabular}

Note: * $-p<0,05$ compared with the control group; ** $-p<0,05$ if compared with the experimental group. 
At the same time, reflecting the severity, course and intensity of general periodontitis and intensity of inflammatory and degenerative processes in periodontal tissues, cellular composition of gums in cytograms was divided into four types. The first type is characterized by presence of intermediate and parabasal cells stained by Romanovskyy-Himza, with smaller size compared to intermediate. These epithelial layers correspond to deep ribbed cells in histological sections. They are larger in size if compared to basic cells and have an elongated shape. The core is large, well-countered, vesicular, with small diffuse located chromatin inclusions and relatively wide rim of basophilic cytoplasm. The lack of hematogenous cells row is noted. It is obvious that this cellular composition obtained in cytograms proves evidence of proliferation, i.e. reproduction of parabasal cells and specific prognostic criterion of generalized periodontitis of initial stage.

In the second type of cytogram there were individual representatives of rod flora with advantage of basophilic intermediate (azur positive) epithelial cells from cytological smears of gums. They have cubic, rectangular or polygonal form; cytoplasm contains azure-positive granules. The core is round, sometimes oval. The trend towards clusters of cells location of this class is noted. Intermediate cells in histological sections correspond to ribbed parts of surface layer. These intermediate cells are found in normal and mostly without elements of cytopathology. We argue that this type of cytograms proves chronic generalized periodontitis.

In the third type of cytogram there were cytograms of intermediate cells staining by Romanovskyy-Himza. They have azure-positive cytoplasm nucleus centric located. The cytoplasm is elongated. The cells are usually located in clusters. The change in the microbial composition is determined that in this type of cytogram is represented mainly by cocci that adhere on the surface of epithelial cells.

In addition to the above mentioned epithelial cells in cytogram of the third type of the examined people there are segmented leukocytes, which are split into 3-4 segments core also there are isolated mononuclear leukocytes or young white blood cells it suggests a redistribution pool of myeloid cells in response to the presence of foci of active inflammatory process that causes the process of differentiation of leukocytes available. Present cytological pattern characterizes pointer of general periodontitis in the examined people.
In the fourth type of cytograms there were single basal epithelial cells. During staining by Romanovskyy-Himza, if compared with basal cells of periodontal pockets they have relatively smaller and more rounded shape of cytoplasm. The core is round-shaped and has a narrow rim of cytoplasm. Basal epithelial cells are surrounded by segmented leukocytes.

The predominant cells in the fourth type of cytograms are the cells of intermediate layer, their cytological organization corresponds a lot to the class affiliation and level of differentiation, a pathogenic microflora is evidenced as well as the cells of inflammatory response. Superficial cells are also verified.

A powerful microbial composition initiates further necrobiotic processes in epitheliocytes and in segmented leukocytes. Due to phagocytosis, the destruction of the cytoplasm of segmented leukocytes occur, it is 'incomplete phagocytosis'.

Coccal microflora adheres not only on the surface of epithelial cells, but also on the surface of segmented leukocytes. Along with haematogenous cells in cytograms predominantly coccoid flora and single thread of pseudomycelia of Candida fungi are evidenced.

So cytograms of the fourth type present the increase of phagocytic reaction of segmented leukocytes, and as a result of incomplete phagocytosis formation of 'pus cells' occurs. During phagocytosis of leukocytes specific changes in the form of restructuring of its nuclear takes place, while in epitheliocytes of gums a necrobiotic process occurs primarily in the cytoplasm and in the nucleus.

The described type of cytogram corresponds to the clinical manifestations of general periodontitis with abscess formation.

The emergence of epithelial cells with signs of irritation in cytograms is one of cytological signs of periodontal pathology in young people. These cellular elements belong to the intermediate layer of cells that on histological sections correspond to ribbed surface layers and are characterized by homogenization and vacuolization of the cytoplasm as a manifestation of prolonged bacterial irritation. The vacuolization of epithelial cells cytoplasm in general periodontitis gingival complex proves disorders of water and electrolyte and protein metabolism, causing the changes in colloid-osmotic pressure in the cell. The violation of cell membrane permeability, accompanied by their collapse is also significant. It leads to lysosomal membranes labilization with activation of their hydrolytic 
enzymes that break intramolecular connection with water. In fact, these changes are a manifestation of early cell focal necrosis [5].

At the same time the increase in the volume of epithelial cells was evidenced, the cytoplasm is full of vacuoles, containing a clear liquid. The core is moved to the periphery of the cell, sometimes are vacuoles or core shrinks. Later disintegration of ultra-structural elements of cells occurs and it overflows with water. These changes are the features of the intermediate stage of cellular metabolism disorders caused by inflammation and preceding cell cytotransformation into balloons filled with fluid. Such changes characterize balloon cell degeneration.

Analysing the cytogram we can reach the conclusion that unlike previous smear from epithelial gums it has the greater degree of destruction accompanied by cariopiknosis and

\section{References}

1. Bainbridge BW. Porphyromonas gingivalis lipopolysaccharide: an unusual pattern recognition receptor ligand for the innate host defense system. Acta Odontol Scand 2001; 59: 131-138.

2. Bourgeois D. Epidemiology of periodontal status in dentate adults in France. J. Periodontal. Res 2007; 42, (№ 3): 219-227.

3. Bykov VL. Functional morphology of epithelial barrier of oral mucosa. Dentistry, 1997; (№ 3): 12-17.

4. Hasiuk NV. Morphofunctional organization of gums in normal state and in case of inflammation. Thesis of Dis. for PhD: specialty 14.03.09 Histology, Cytology, Embryology, 2009; 19.

5. Hasiuk NV. Ultrastructural arrangement of gum furrow epithelial. Bulletin of Ukrainian Medical Dental Academy: Topical Matters of Contemporary Medicine, 2013; 13 (42): 189-191.

6. Grudianov AI. Periodontal diseases. M : Medical News Agency, 2009: 336.

7. Kornman KS. The "innovator's dilemma" for periodontists. J. Periodontol 2010; 81 (№ 5): 646-649. kariorexis of nucleus and cytoplasm homogenization. Horny scales in cytograms decreased in comparison to their quantitative composition in cytograms of people with health periodontium. They are mainly eosinophilic, of polygonal shape, characterized by no clear contours.

\section{Conclusions}

The changes in the gums in the course of generalized periodontitis are subject to rather wide limits, which depend on the clinical course and exogenous factors. Analysis of cytograms gave the opportunity for consideration of general periodontitis in the examined patients due to the absence of adequate therapy as automotive continuous process. Its components (inflammatory infiltrative and destructive) may decrease after the effective treatment and reactivate during the exacerbation of inflammatory process under the influence of pathogenic factors such as microbial, hygienic and immune.

8. Kononen E. Population-based study of salivary carriage of periodontal pathogens in adults. J. Clin. Microbiol 2007; 45, (№ 8): 2446-2451.

9. Schmalz G. Release of prostaglandin E2, IL-6 and IL-8 from human oral epithelial culture models after exposure to compounds of dental materials. Eur. J. Oral. Sci. 2000; 108: 442-448.

10. Schnare M. Toll-like receptors: sentinels of host defence against bacterial infection. J. Allergy Immunol 2006; (139): 75-85.

11. Shi D. Inflammatory bowel disease requires the interplay between innate and adaptive immune signals. Cell Res. 2006; (16): 70-74.

12. Van Dyke TE. Inflammation and factors that may regulate inflammatory response. J. Periodontol. 2008; 79 (8): 1503-1507.

13. Vitkov LV. Bacterial internalization in periodontitis. Oral Microbiol. Immunol. 2005; 20 (5): 317-321. 